\title{
汉英翻译中比喻的翻译研究一一张培基《英译中国现代散文选》 为例
}

\author{
朱蕾 \\ 江西财经大学外国语学院 \\ DOI:10.32629/er.v3i1.2387
}

[摘 要] 比喻作为一种重要的修辞手法,在汉语中的使用由来已久。比喻赋予语言以灵气和色彩,给读者留有想象、体会的空间。进行汉英翻 译时,译者需要兼顾汉语比喻内涵和英语表达习惯。本文以张培基《英译中国现代散文选》为例,总结了不同类型的比喻的翻译策略及方法 [关键词] 比喻; 直译; 意译; 译文读者

张培基《英译中国现代散文选》精选中国现代散文名篇进行翻译, 出 版以来受到众多学子、学者的推崇, 也为汉英翻译研究提供了丰富的素材。 比喻是一种将两个不同事物由某一相似点联系在一起的修辞手法。比喻让 语言产生了联想意义, 让语言更加鲜活、形象、生动, 增强了表达效果。各 种巧妙的比喻丰富了语言的内涵, 也进一步丰富了语言文化。比喻的英译 可根据不同情况采用不同的翻译策略及方法, 张培基《英译中国现代散文 选》中涉及众多比喻的巧妙英译, 值得深入研究、学习和借鉴。

\section{1 比喻的认知}

1. 1 比喻的特征

比喻主要由本体 (喻体所依附的对象) 和喻体 (形容本体的术语) 组成。 比喻在词语旧义的基础上创造出新义, 新义与旧义往往互相联系、存在相 似性。另外, 比喻是一种语言使用现象, 需要结合特定语境进行分析。单独 的词不能构成比喻, 也不具有分析的价值。

\section{2 比喻的功能}

比喻的使用丰富了语言的多样性, 增强了语言的魅力。比喻的功能主 要有以下几点: (1) 比喻让表达更加生动且富有感染力, 并引起读者的联想 与想象, 让人印象深刻; (2) 比喻能化抽象为具体, 增强语言的直观性, 让人 在已有的主观经验的基础上理解没有经历过或看到过的事物; (3) 比喻可 以用简洁的语言表达某些复杂或难以明言的思想, 化深奥为浅显, 增加语 言的易懂性。

\section{2 比喻的翻译策略及方法}

2.1 以源语为导向的翻译

以源语为导向的翻译遵循异化的翻译策略, 让读者向作者靠拢。比喻 的异化翻译有助于保存源语的风格特色, 体现文化内涵。比喻的异化翻译 往往采取直译手法, 取其喻意进行翻译, 保留了原文本的修辞效果。

人类具有共有的非文化知识, 即不同民族的非相互习得的知识以及某

的理论指引与技术支撑, 努力为家校共育提供更加丰富饱满的精神食粮, 合理引领家校共育和谐有序发展。每学年学校可评选一批家校共育模范家 庭, 予以表彰, 这样可以充分发挥榜样的力量, 带领全部家长全力以赴投入 家校共育的工作中

\section{6 建立网上 “家校共育讲堂”}

学校借助校园网和学校网站, 建立网上 “家校共育讲堂”, 和家校共育 专栏, 将优秀的家庭教育讲座推荐给家长, 在专栏上发送有关家庭教育的 文章, 把当前先进的教育理念、方法系统性地呈现给家长, 为学生家校共育 提供科学的理论指导, 促进家校共育的健康发展。

3 结束语
些认知过程是相似的。汉语中有些比喻在英语中有相似的含义, 或能带来 相似的体验或感受, 采用直译法不但不会影响译文读者的理解, 还能保留 原比喻的形式特色和修辞效果。如:

例1：骆驼的峰就是一座拱桥, 它沟通了东方和西方的文化….... ${ }^{[1]}$

译文: The camel's hump symbolizes an arch bridge linking up the cultures of East and West. ${ }^{[2]}$

“桥”此处指的是一种“纽带”, 《牛津高阶英汉双解词典》对“bridge” 一词有着近乎相同的定义 “a thing that provides a connection or contact between two different things”。汉译英时, 重现这类比喻意象 丝毫不会影响译文读者对译文的理解。

例2: 巷, 是城市建筑艺术中一篇飘逸恬静的散文, 一幅古雅冲淡的图画。 ${ }^{[3}$

译文: The lane, in terms of the art of urban architecture, is like a piece of prose of gentle gracefulness or a painting of classic elegance and simplicity.

无论是在中国还是在西方国家，“散文”和 “图画” 都能带给人以美 的体验和享受, 译文读者可在这种固有的思维基础上进行理解。由于人类 对这类事物的感知是相同或相似的, 直译不会造成语言上的障碍, 译文读 者稍加联想即可感知原作意境。

有些文学作品中运用意象的罗列来表达浓烈的情感或增强语言的表 现力与审美情趣。文学翻译注重翻译的文学性, 译文应尽量再现原作艺术、 意境, 给读者以近似于阅读原文的美的感受。

例3: 比起北国的秋来, 正像是黄酒之与白干, 稀饭之与馍馍, 鲇鱼之与 大蟹, 黄犬之与骆驼。

译文: Southern autumn is to Northern autumn what yellow rice wine is to kaoliang wine, congee to steamed buns, perches to crabs, yellow dogs to camels.

总之, 在互联网的环境条件下, 搭建家庭与学校之间全程、全员及全方 位育人的立交桥, 使交流更为畅通高效, 使家庭与学校的关系更加和谐, 是 促进家校共创与教育健康发展的新颖平台。

[参考文献]

[1]黄盈.浅析初中语文教学中的多元化教学方法 [J].小作家选 刊,2015(26):146-147.

[2]刘荣信.对初中语文教学中的多元化教学方法探究 [J]. 课外语 文,2015(24):119.

[3]胡强.多元化教学策略在初中语文教学中的有效实施策略探究 [J]. 文理导航(上旬),2015(04):4-5 
“黄酒”、“白干”、“稀饭”、“馍馍”、“鲇鱼”、“大蟹”、“黄犬”、“骆 驼” 这八个意象铺排而下, 让人避想、回味。直译有助于保留原文的文学 性和审美价值, 同时, 适度留白给译文读者留下更大的思索空间。

汉语中有些比喻引用了外国的历史典故或文学作品中的意象。原意象 家喻户晓, 随着时间的推移, 原意象的译本也已受到译文读者的认可和接 受。在翻译这类比喻时, 可参考其出处, 直译更符合接受习惯。

例 4: 我在那时候当然是 “丑小鸭”, 自己也是知道的, 但是终不以此 而减灭我的热情。 ${ }^{[5}$

译文: Of course I knew then I was nothing but an “Ug1y Ducking”, but that didn' $\mathrm{t}$ damp down my passion.

“丑小鸭”来源于丹麦作家安徒生创作的童话, 已被翻译成多种文字, 广为流传。参照其英文译本, 直译更易受到西方读者的认可和接受。

\section{2 以目标语为导向的翻译}

以目的语为导向的翻译遵循归化的翻译策略, 把源语本土化, 译文的 表达尽量符合目的语读者的接受习惯。比喻的归化翻译常常采用意译手法, 取其本意进行翻译, 从而保证译文的易懂性。比喻的意译重在合理阐述原 隐喻的内涵而非形式, 帮助译文读者更好地理解原文。

翻译的重要目的在于打破不同文化背景下语言的障碍, 促进两种语言 之间信息的传递。汉英两种语言之间存在文化差异, 汉语中有些意象或喻 体形象无法在英语中引起同等或相近的感受和联想。这类比喻常出现在人 们的日常口语中并逐渐成为约定俗成的表达, 或称惯用语。这类比喻的核 心含义在其喻义, 直译其字面意义不仅没有太大意义, 还很有可能造成译 文读者的错误解读。如:

例5: 穷困时, 就一个人跑去马路上喝西北风…... ${ }^{[6]}$

译文: When I' m broke, I' 11 go strolling around the streets alone on an empty stomach.

“喝西北风” 是常见的汉语惯用语, 喻指没吃饭, 饿肚子。这一隐喻并 不能给译文读者带来近似的联想, 采取意译法更加简明扼要、清晰明了。

另外, 中国有很多耳熟能详的典故用作比喻可以使语言具有艺术性且 不会造成中国读者的阅读障碍, 但是外国读者并不了解该典故的内涵, 译 者也很难在译人语中找到直接对应的翻译。翻译这类隐喻时应将其置于特 定语景中理解, 重在阐述原语内涵。如:

例6: 得势的一只雄鸡不消说要欺负它, 便连那些娥皇女英们也不把它 看在眼里。 ${ }^{[7]}$

译文: Not only was he bullied by the stronger opponent, he was also snubbed by the three females.

“娥皇女英” 是中国古代神话传说中的两位女性, 在此处指前文提到 的三只母鸡。保留原喻体形象会导致午余信息过多, 导致译文读者阅读译 文时存在障碍。译者需要抛弃原语形式, 重在阐述喻意以确保原文信息的 有效传递。

还有些比喻蕴含了作者所处时代背景下的社会文化, 因而含有特殊含
义。这类比喻具有很强的针对性, 需要结合上下文分析其所指含义。这类 比喻的直译会让人云里雾里, 不知所云; 意译更有助于读者理解原文。如:

例 7: 现在有些刊物, 派人到北京上海各出跑, 约文章, 等米下锅….... ${ }^{[7]}$

译文: Some newspapers and magazines are so starved of contributions that they have to send out people to various places like Beijing and Shanghai to canvass for support.

“等米下锅”一般用来形容生活困难或情势极窘迫, 此处喻指刊物闹 稿荒。结合上下文, 采用意译手法, 重在展示原文背景下比喻的所指含义。

\section{3 删除或省略}

为了加强语言修辞效果, 汉语中经常会有语义重复的情况。英语则倾 向于避免重复, 注重用词精确。汉译英时如果完全按原句进行翻译, 译文会 显得累赘。翻译时可删除或省略一些语义累赘的词句, 剔除穴余信息, 力求 语言简洁、精炼, 从而满足译文读者的阅读习惯。如:

例8: 弓儿似的新月, 挂在树梢。

译文: The crescent new moon looked as if hanging on the tips of the trees.

例9: ……眉痕的新月已经出现在鲜红的云缝里了。 ${ }^{[6]}$

译文: $\cdots$ the crescent new moon was already peeping out from behind the scarlet clouds.

“crescent”一词在英文中就有 “新月形” 的意思, 足以让读者体会 到原作者所描绘的月亮。“弓儿” 和 “眉痕” 两处比喻的省略避免了重复 啰嗦, 使译文更加精简流畅。

\section{3 结语}

译者要基于对原文的理解, 捕捉原文比喻的审美意图及其艺术性, 并 在保证译文接受性和易懂性的基础上, 最大限度地传达原比喻的修辞效果 和语言魅力。本文结合张培基《英译中国现代散文选》中比喻的英译, 总 结不同情况下比喻的翻译策略及方法, 望能提供可供借鉴的经验技巧。不 足之处, 敬请指正。

\section{[参考文献]}

[1]程同春.英语隐喻的思考与翻译[J].中国科技翻译,2005,(5):36-40. [2]戴卫平.词汇隐喻研究[M].广州:世界图书出版公司,2014:1-20.

[3]胡壮麟.隐喻翻译的方法与理论 [J]. 当代修辞学,2019,(4):1-8.

$[4]$ 吴礼权.现代汉语修辞学 [M].上海:复旦大学出版社,2016:78-102.

[5]曾剑平.汉语语义重复词句的省译[J].中国科技翻译,2018,(11):37-40.

[6]张培基.英译中国现代散文选(一)[M].上海:上海外语教育出版 社,2007:94-274.

[7]张培基.英译中国现代散文选(二)[M].上海:上海外语教育出版 社,2007:58-304.

作者简介:

朱蕾(1996--)女,汉族,湖北黄冈人,在读研究生,研究方向：翻译。 